\title{
Rezidivierende respiratorische Papillomatose: Aktuelles zu Diagnose und Therapie
}

Christine Langer, Claus Wittekindt, Gregor Wolf

Die rezidivierende respiratorische Papillomatose (RRP) - ausgelöst durch das humane Papillomavirus (HPV) - ist durch warzenartige Veränderungen der Atemwege gekennzeichnet. Der klinische Verlauf ist variabel, die Diagnosestellung erfolgt nicht selten wesentlich später als der Symptombeginn, insbesondere bei Kindern. Die Behandlung wird individuell festgelegt und umfasst meist Operationen zur Tumorkontrolle und zum Erhalt der Stimmfunktion.

\section{Einführung}

Die rezidivierende respiratorische Papillomatose (RRP) zeichnet sich durch das Auftreten von warzenartigen Veränderungen der oberen, seltener auch der unteren Atemwege aus. Auslöser der Erkrankung ist das humane Papillomavirus (HPV). Der klinische Verlauf der Erkrankung ist sehr variabel, und die Diagnosestellung erfolgt nicht selten mit erheblichem Intervall zum Symptombeginn, insbesondere bei Kindern. Das therapeutische Konzept ist regelhaft individualisiert und beinhaltet überwiegend Operationen zur Tumorkontrolle und zum Erhalt der Stimmfunktion. Dieser Beitrag gibt einen Überblick über die Genese und Epidemiologie der Erkrankung, fasst die diagnostischen sowie therapeutischen Möglichkeiten zusammen und stellt die aktuelle Literatur zum Thema dar.

\section{Epidemiologie}

Die Erkrankung weist eine bimodale Altersverteilung bezogen auf den Zeitpunkt der Erstmanifestation auf:

- Die juvenile Form (Juvenile Onset Respiratory Papillomatosis, JORPP) ist definiert durch ein erstmaliges Auftreten vor dem 12. Lebensjahr und manifestiert sich meist zwischen dem 2. und 4. Lebensjahr [1].

- Die adulte Form (Adult Onset Respiratory Papillomatosis, AORPP) zeigt ihre klinische Erstmanifestation in der 2. bis 4. Dekade [2].

\section{Merke}

Die juvenile Form der RRP wird erstmalig vor dem 12. Lebensjahr diagnostiziert. In höherem Lebensalter auftretende Fälle sind per Definition adulte Formen der Erkrankung.

Die Inzidenz bei JORPP wird mit 1,9 bis 4,2 pro 100000 angegeben [3]. Es handelt sich damit um die häufigste benigne Neubildung des Larynx und eine der häufigsten Ursachen einer Dysphonie im Kindesalter. Eine Geschlechtsprävalenz besteht nicht. Das Auftreten im frü- hen Kleinkindalter scheint häufig mit einem aggressiven Krankheitsverlauf assoziiert zu sein [4], [5]. Hier finden sich vermehrt Formen mit einem Befall der unteren Atemwege bis hin zur endobronchialen und pulmonalen Aussaat und sogar vereinzelt letalen Verläufen.

Merke

Ein früher Erkrankungsbeginn bedeutet ein höheres Risiko für schwere Krankheitsverläufe mit häufigerem Befall von Bronchialbaum und Lunge.

Bei AORPP dominiert das männliche Geschlecht mit $1: 2$ bis 1 : 4 Fällen. Die Inzidenz ist mit 1,8 bis 3,8 zu 100000 ähnlich wie bei der juvenilen Form. Der klinische Verlauf ist in den meisten Fällen jedoch weniger aggressiv und das Auftreten einer bronchopulmonalen Beteiligung deutlich seltener [6].

\section{Ätiologie}

\section{Erreger}

Auslöser der RRP ist das Humane Papillomavirus (HPV). Es handelt sich dabei um ein kleines, unbehülltes DNA-Virus aus der Gruppe der Papillomaviridae. Mittlerweile sind mehr als 200 Varianten des Virus bekannt, welche ausgehend von ihrem onkogenen Potenzial in Low-Risk-, Intermediate-Risk- und High-Risk-Erreger differenziert werden [7].

Der Nachweis humaner Papillomaviren in respiratorischen Papillomen erfolgte erstmals 1980. Die seither vorgenommene Subtypisierung ergab HPV-6 und HPV-11 als häufigste Verursacher der RRP:

- HPV-11 findet sich in 50-60\% der RRP und ist gekennzeichnet durch einen eher aggressiven klinischen Verlauf.

- Die in 20-40\% durch HPV-6 verursachte RRP verläuft weniger aggressiv, beispielsweise gekennzeichnet durch seltener notwendige operative Eingriffe [8]. 


\section{Merke}

Der Krankheitsverlauf ist abhängig vom nachgewiesenen HPV-Subtyp. HPV-6 verursacht mildere Verläufe mit weniger häufigem Therapiebedarf, während ein Nachweis von HPV-11 mit aggressiveren klinischen Verläufen einhergeht.

Die im Zusammenhang mit malignen Erkrankungen des Oropharynx und des Zervikalkanals in den vergangenen Jahren vermehrt untersuchten HPV-Subtypen 16, 18, 31 und 33 kommen in deutlich geringerer Inzidenz auch in respiratorischen Papillomen vor [9]. Es existieren wenige Fallberichte über spontane maligne Transformationen bei RRP. Hier finden sich die genannten HPV-Hochrisikogruppen statistisch häufiger, aber nicht ausschließlich. Unabhängige Risikofaktoren wie ein stattgehabter oder aktiver Nikotinabusus oder eine vorangegangene Strahlentherapie des oberen Atemwegs haben wahrscheinlich keinen Einfluss auf das Entartungspotenzial [10].

Humane Papillomaviren sind streng artspezifisch und weisen auch einen zellulären Tropismus auf. Die eine RRP verursachenden HPV-Subtypen der Gruppe $\alpha$ infizieren daher ausschließlich Schleimhautzellen, und das Virus ist intraepithelial lokalisiert. Die intraepitheliale Lage ist verantwortlich für eine deutlich erschwerte immunologische Erkennbarkeit des Erregers. Papillome finden sich häufig an der Transitionszone zwischen Platten- und Flimmerepithel, somit häufig intralaryngeal. Dies entspricht der Transitionszone der Cervix uteri bei der genitalen HPV-Infektion. Ungeklärt ist der Entstehungsmechanismus der Erkrankung aus der Infektion, da die Infektion mit HPV ubiquitär ist, aber nur in den seltensten Fällen zur Erkrankung RRP führt. Hier scheinen immunologische Defekte eine Rolle zu spielen, die bislang jedoch nicht lokalisiert werden konnten. Somit ist wahrscheinlich - weniger als die Infektion - die Prädisposition des Wirts für das Auftreten der Erkrankung bedeutsam.

\section{Infektionsweg}

Der genaue Mechanismus der Übertragung ist nicht bekannt. HPV kann in der einfachen Zellkultur nicht vermehrt werden, was Untersuchungen zur Pathogenese erschwert. Epidemiologische Untersuchungen legen jedoch mögliche Infektionswege nahe. Die Kenntnis einer erhöhten Prävalenz von maternalen anogenitalen Papillomen bei JORRP lässt z. B. Rückschlüsse auf eine mögliche perinatale Übertragung zu [11]. Auch die Tatsache, dass Kinder mit JORRP vaginal entbunden worden sind, stützt die Theorie der vertikalen Übertragung von Mutter zu Kind im Geburtskanal. Postpartal kann HPV wochenlang in der pharyngealen Schleimhaut von Neugeborenen, deren Mütter an genitalen Kondylomen erkrankt sind, nachgewiesen werden [12].

Dennoch ist die Inzidenz bei JORRP verglichen mit der Häufigkeit genitaler Kondylome sehr gering. Geschätzt erkran- ken nur 7 von 1000 Neugeborenen von Müttern mit genitalen Kondylomen an einer JORRP [13]. Folglich ist die Sectio caesarea auch bei Kondylomen in der Schwangerschaft nicht generell empfohlen. Als weitere Risikofaktoren für JORRP gelten ein mütterliches Alter unter 20 Jahren und Erstgeburtlichkeit [14]. Letzteres wird am ehesten durch einen üblicherweise ausgedehnteren Geburtsverlauf und den daher verlängerten Kontakt zum Geburtskanal erklärt. Diskutiert werden weiterhin die Immunität des Kindes, die Viruslast und lokale Traumata der Schleimhaut, z. B. im Rahmen einer Intubation.

Mit den oben genannten Mechanismen kann die Erkrankung AORRP jedoch nur wenig hinreichend erklärt werden. Da humane Papillomaviren eine Persistenz in der Basalmembran ohne klinische Hinweise verursachen können, ist eine Aktivierung des Virus dennoch denkbar [15]. Auch eine sexuelle Übertragung des Virus wird immer wieder diskutiert, da bei an AORPP erkrankten Patienten mehr Sexualpartner und häufigerer Oralverkehr empirisch erfasst werden [16].

\section{Merke}

Der Übertragungsweg bei RRP ist nicht eindeutig geklärt. Epidemiologische Untersuchungen legen eine peripartale Infektion im Geburtskanal oder sexuelle Übertragungswege nahe.

\section{Klinik}

Das klinische Erscheinungsbild bei JORRP und AORRP ist abhängig von der primären Lokalisation der Papillome. Erstes Symptom ist in den meisten Fällen eine persistierende oder progrediente Heiserkeit, zu der im wochenoder monatelangem Verlauf insbesondere bei der JORRP Dyspnoe mit inspiratorischem oder biphasischem Stridor durch Verlegung der Atemwege kommen kann. Bei JORRP erfolgt die Diagnosestellung als Folge der erschwerten Untersuchbarkeit im Kindesalter kombiniert mit der Seltenheit der Erkrankung häufig erst im fortgeschrittenen Stadium bei Obstruktion der oberen Atemwege. Das klinische Bild umfasst auch Fälle von Gedeihstörungen durch Dysphagie oder kompletter Verlegung der Atemwege bei initial als Pseudokrupp oder asthmatische Erkrankung fehlgedeuteten Symptomen [17].

Prinzipiell ist ein Befall des gesamten oberen und unteren Atemwegs beginnend in der nasalen Schleimhaut bis in die Peripherie der Lunge möglich. Die häufigste Lokalisation ist jedoch mit 95\% der Endolarynx. Gehäuft treten Papillome auch am Limen nasi, am weichen Gaumen und im Nasopharynx auf [18]. Bei einem laryngealen Befall finden sich oft zusätzlich Läsionen an der laryngealen Epiglottis. Ein trachealer oder pulmonaler Befall ist dagegen selten und mit einer sehr schlechten Prognose quoad vitam assoziiert [19]. 


\section{FALLBEISPIEL}

Juvenile Onset Respiratory Papillomatosis (JORPP) Ein 9 Monate alter Junge wird intubiert und beatmet aus einer externen Kinderklinik verlegt. Nach Angaben der Mutter weise das Kind seit seinem 6. Lebensmonat eine raue Stimme auf. In den letzten Tagen sei es zu zunehmender Atemnot gekommen, weshalb Mutter und Kind mit Verdacht auf Pseudokrupp an die nächstliegende Kinderklinik verwiesen wurden. Bei respiratorischer Verschlechterung mit in- und exspiratorischem Stridor wurde dort die Intubation des Kindes notwendig. Bronchoskopisch zeigte sich eine Atemwegsverlegung der Supraglottis und Glottis suspekt auf eine ausgedehnte respiratorische Papillomatose. Es erfolgte eine sofortige laserassistierte mikrolaryngoskopische Abtragung der die Glottis verlegenden Papillome unter Schonung der laryngealen Strukturen, insbesondere der vorderen Kommissur ( $\triangleright$ Abb. 1). Dabei stellte sich auch ein subglottischer Befall dar, welcher ebenfalls abgetragen wurde. Im postoperativen Verlauf von bislang 1 Jahr wurden regelmäßige Mikrolaryngoskopien wegen erneuter Verlegung des Atemwegs im 2- bis 3-monatigen Abstand notwendig. Histopathologisch zeigten sich Papillome mit Nachweis von HPV 11. Bei der weitergehenden Anamnese gibt die Mutter an, sich bis kurz vor der (vaginalen) Geburt des Kindes aufgrund von genitalen Kondylomen in chirurgischer Therapie befunden zu haben.

Aus histologischen Untersuchungen ist bekannt, dass die Prävalenz der HPV-DNA in der trachealen verglichen mit der laryngealen Schleimhaut gleich ist [20]. Die dagegen deutlich häufigere Papillomatose des Larynx weist auf mögliche zelluläre immunologische Mechanismen hin, die zur differenten Wahrscheinlichkeit der klinischen Manifestation beitragen. Weiterhin spielt das Fehlen einer epithelialen Transitionszone kaudal des subglottischen Abhangs eine Rolle.

\section{Merke}

Bei RRP ist zu 95\% die laryngeale Schleimhaut befallen. Prinzipiell kann aber der gesamte obere Aerodigestivtrakt betroffen sein. Ein Befall von Bronchien und Lunge ist mit einer schlechten Prognose assoziiert.

\section{Diagnose}

Die Verdachtsdiagnose RRP wird in der Regel starr oder flexibel endoskopisch gestellt. Dabei zeigen sich die typischen, oft gruppierten warzigen Veränderungen mit charakteristischer Gefäßzeichnung ( $>$ Abb. 2).

Der Nachweis der Erkrankung erfolgt durch Biopsien aus der Läsion im Rahmen einer Mikrolaryngoskopie und his-

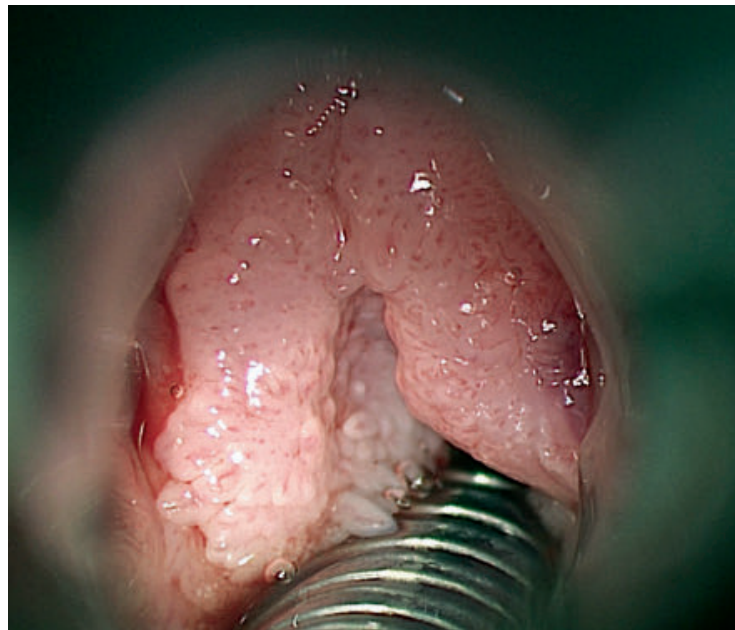

Abb. 1 Juvenile Papillomatose mit Verlegung des Atemwegs bei einem 9 Monate alten Kind.

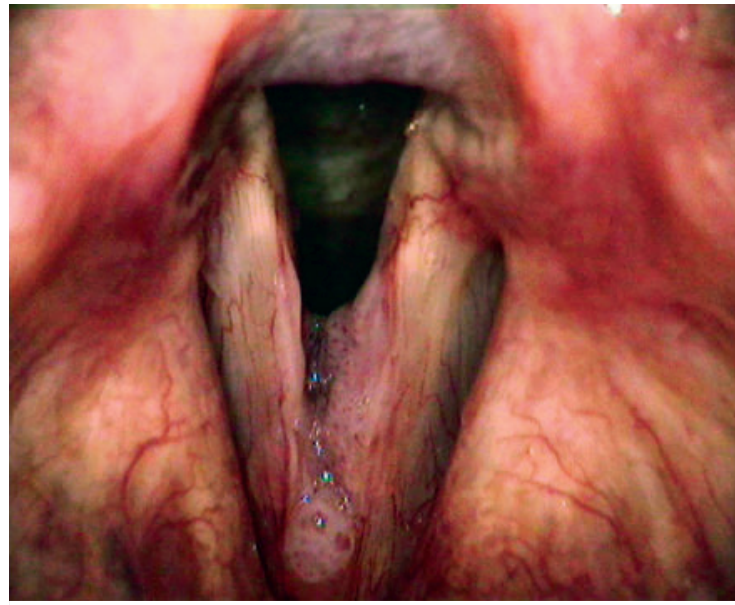

Abb. 2 Lupenlaryngoskopische Aufnahme einer laryngealen RRP mit typischer verruköser Veränderung der Stimmlippe (Quelle: Brockmann-Bauser M, Bohlender J. Larynxpapillomatose. In: Schrey-Dern D, Lauer N, Hrsg. Praktische Stimmdiagnostik. 1. Auflage. Stuttgart: Thieme; 2014).

tologischer Untersuchung mit molekularem Nachweis der HPV-DNA im befallenen Gewebe. Die präoperative Verbesserung der Ausbreitungsdiagnostik kann durch Inhalation von 5-Aminolävulinsäure erfolgen. Hierdurch zeigen die Papillome eine rötliche Fluoreszenz im Gegensatz zur blau erscheinenden gesunden Schleimhaut, sodass auch kleine Läsionen besser sichtbar werden [21].

Die klinische Bewertung des Schweregrads der Erkrankung kann beispielsweise mit der Erfassung des sogenannten Derkay-Scores erfolgen. Dabei wird vom Operateur die Ausbreitung der Papillome auf die einzelnen Regionen des Larynx bewertet und summiert [22]. Es kann eine Gesamtpunktzahl von 75 erreicht werden, eine höhere Punktzahl ist z. B. mit einem kürzeren interoperativen Intervall assoziiert ( Tab. 1). 
- Tab. 1 Bewertung der Ausbreitung durch Einschätzung des Operateurs nach Derkay.

\begin{tabular}{|c|c|c|c|}
\hline Struktur & Bewertung & Rechts & Links \\
\hline \multicolumn{4}{|l|}{ Larynx } \\
\hline Epiglottis laryngealis & () & - & - \\
\hline Epiglottis lingualis & () & - & - \\
\hline $\begin{array}{l}\text { Aryepiglottische } \\
\text { Falte }\end{array}$ & - & () & () \\
\hline Taschenfalte & - & () & () \\
\hline Stimmlippe & - & () & () \\
\hline Arytaenoide & - & () & () \\
\hline vordere Kommissur & () & - & - \\
\hline hintere Kommissur & () & - & - \\
\hline Subglottis & () & - & - \\
\hline \multicolumn{4}{|l|}{ Trachea } \\
\hline oberes Drittel & () & - & - \\
\hline mittleres Drittel & () & - & - \\
\hline unteres Drittel & () & - & - \\
\hline Hauptbronchus & - & () & () \\
\hline Tracheostoma & () & - & - \\
\hline \multicolumn{4}{|c|}{ Andere Lokalisationen } \\
\hline Nase & - & () & () \\
\hline Gaumen & - & () & () \\
\hline Pharynx & - & () & () \\
\hline Ösophagus & () & - & - \\
\hline Lunge & () & - & - \\
\hline Weitere & () & () & () \\
\hline
\end{tabular}

\section{Therapie}

Die Therapie bei RRP ist nicht einheitlich. Standard ist die chirurgische Resektion im Rahmen einer Mikrolaryngoskopie bei Befall der Stimmlippen. Da humane Papillomaviren nicht nur in makroskopisch sichtbaren Papillomen vorhanden sind, sondern sich auch in der benachbarten Schleimhaut nachweisen lassen, kann eine „radikale“ chirurgische Therapie meist nicht zielführend sein. Das Virus bleibt auf das Epithel begrenzt, daher kann auch eine tiefe Resektion offensichtlich keinen Vorteil in der Therapie erbringen.

Der funktionelle Organerhalt steht somit im Vordergrund. Hierzu gehört neben der Stimmfunktion bei Befall des Larynx auch das Freihalten des Atem- und Schluckwegs. Ein rekurrentes Auftreten der Symptome nach operativer Therapie der Papillome entspricht dem typischen Verlauf bei RRP. Daher sind wiederholte Abtragungen regelhaft notwendig. Die Anzahl und die Art der notwendigen Eingriffe bestimmen nicht unwesentlich das funktionelle Ergebnis. Daher ist grundsätzlich ein langes interoperatives Intervall wünschenswert. Dies ist insbesondere bei Kleinkindern der Fall, da hier die Notwendigkeit operativer Eingriffe deutlich häufiger ist als in höherem Lebensalter. In einer umfangreichen Registerstudie zeigte sich, dass Kleinkinder mit einer Erstmanifestation vor dem 3. Lebensjahr ein vielfach erhöhtes Risiko hatten, sich mehr als 4 operativen Eingriffen pro Jahr unterziehen zu müssen, als Patienten mit Diagnosestellung in höherem Alter [23].

In einer empirischen Untersuchung der British Association of Paediatric Otorhinolaryngology wurden die bevorzugten chirurgischen Verfahren für die Abtragung der Papillome erfragt [24]. Die am häufigsten genannten Verfahren waren die operative Abtragung mittels $\mathrm{CO}_{2}$ - und KTP-Laser gefolgt von der Verwendung des Microdebriders und mikrochirurgischen, kalt schneidenden Techniken.

\section{Merke \\ Ziel einer chirurgischen Therapie bei RRP ist der funk- tionelle Organerhalt.}

Die Tumorkontrolle bei Papillomen kann grundsätzlich kalt (Schere oder Debrider) oder heiß (Laser oder elektrische Thermodestruktion) erfolgen.

\section{Microdebrider}

Sogenannte Shaver wurden ursprünglich aus orthopädisch-arthroskopischen Instrumenten entwickelt und werden seit den 1990er Jahren auch in der sinunasalen Chirurgie verwendet. Die weitere Modifikation und Verfeinerung der Schnittwerkzeuge erlaubt nun eine Anwendung auch in der Mikrolarynxchirurgie. Technisches Prinzip ist eine Kombination aus Absaugen und Exzision mit einem rotierenden Schnittwerkzeug. Papillome werden durch den Sog in die rotierende Klinge gezogen. Auf diesem Wege soll das subpapillomatöse, gesunde Gewebe intakt bleiben. Im Gegensatz zur Laserbehandlung bleiben thermische Schädigungen und Aerosolisierung der HPV-Partikel aus [25], durch die Anwendung unter zu hohen Sog kann es dennoch zum Einsaugen und damit zur Schädigung von gesundem Gewebe kommen. Im angloamerikanischen Sprachraum ist der Microdebrider das am häufigsten verwendete Verfahren zur chirurgischen Behandlung bei RRP [26].

\section{Laser-assistierte Operationen}

Die Energie des Kohlendioxid-Lasers wird durch den Wassergehalt des Gewebes absorbiert und führt zur Ablation der Papillome. In Verbindung mit einem Operationsmikroskop angewandt kann hierdurch eine präzise, blutarme Verdampfung von Papillomen mit limitiertem Schaden des darunter liegenden gesunden Gewebes erfolgen [27]. Die Führung wird durch Mikromanipulatoren erleichtert. Schwer direkt zugängliche anatomische Regionen können durch endoskopisch kontrollierte fiberoptische Lasersysteme erreicht werden [28]. 
Risiken der Laserbehandlung sind neben einer thermischen Schädigung des angrenzenden Gewebes und damit verbundener Narbenbildung die Bildung von Aerosolen bei der Vaporisierung der Papillome, welche HPVDNA enthalten können [29]. Daher wird die Möglichkeit der Übertragung des Virus auf bislang nicht befallene Schleimhäute des Patienten, aber auch auf das medizinische Personal diskutiert. In Konsequenz werden aktuell abgeleitet von der Behandlung genitaler Papillome hygienische Schutzmaßnahmen wie das Tragen eines Mundschutzes mit Aktivkohlefilter für Operateur und OP-Personal empfohlen.

Die vermehrt in der Larynxchirurgie eingesetzten photoangiolytischen Laser erlauben es, selektiv kleinste Gefäße unter Schonung des darüber liegenden Epithels und auch der Lamina propria zu veröden. Zu den photoangiolytischen Lasern gehören der 585-nm-gepulste Farbstoff-Laser, der 532-nm-gepulste Kaliumtitanyl-Phosphat-Laser (KTP-Laser) sowie der Argon-Laser (514 nm) und seit Neuestem auch der sogenannte blaue Laser (445 nm). Wirkmechanismus ist dabei eine selektive Absorption von braunen, roten und schwarzen Farbstoffen. Im Gewebe entspricht dies fast ausschließlich dem (Oxy-)Hämoglobin und führt zur sogenannten Photoangiolyse von Kapillargefäßen, wie sie typischerweise in Papillomen vorkommen. Diese Verfahren können sowohl im Rahmen von Mikrolaryngoskopien als auch kontaktendoskopisch in Lokalanästhesie mit flexiblen Laserfasern durchgeführt werden. Ein deutlicher Vorteil scheint bei diesem Verfahren die Möglichkeit des aggressiveren operativen Vorgehens in der vorderen Kommissur zu sein, die zu längeren interventionsfreien Intervallen führt [30].

\section{FALLBEISPIEL}

\section{Adult Onset Respiratory Papillomatosis (AORPP)}

Ein 78-jähriger Patient stellt sich zur weiteren Diagnostik vor, nachdem er eine langsam progrediente Heiserkeit seit mehreren Monaten bemerkt hat. Zunächst ist ihm nur ein verminderter Tonumfang beim Singen im Chor aufgefallen, später eine eingeschränkte Sprechstimme. Die lupenlaryngoskopische Diagnostik zeigt eine Raumforderung der linken Stimmlippe im vorderen bis mittleren Drittel. Daraufhin wird die mikrolaryngoskopische Resektion der Raumforderung indiziert und $\mathrm{CO}_{2}$-Laser-assistiert durchgeführt ( $>$ Abb. 3 ). Intraoperativ besteht bereits der dringende Verdacht auf eine respiratorische Papillomatose. Weitere Herde kommen nicht zur Darstellung. Die histopathologische Begutachtung ergibt ein Papillom ohne Nachweis von dysplastischen Veränderungen, jedoch mit Nachweis von HPV 11. Im weiteren klinischen Verlauf von nunmehr 5 Jahren zeigt sich eine komplette Remission ohne erneute Manifestation der RRP.
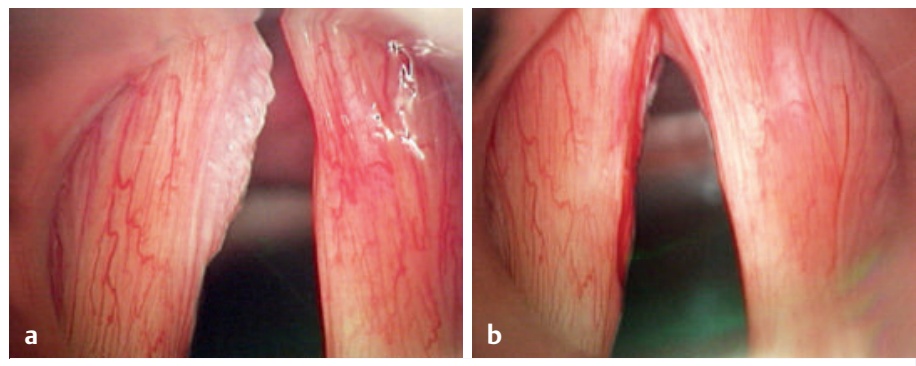

Abb. 3 Mikrolaryngoskopische Sicht auf die Glottis bei AORRP-Befall der linken Stimmlippe. a Präoperativ. b Postoperativ.

\section{Medikamentös-adjuvante Therapie}

Aufgrund des rekurrenten klinischen Verlaufs bei RRP war die Verlängerung des interoperativen Intervalls durch verschiedene medikamentöse Adjuvanzien in den vergangenen Jahren Gegenstand zahlreicher klinischer Untersuchungen. Die Seltenheit und die klinische Variabilität der Erkrankung erschweren jedoch die Durchführung kontrollierter Studien. Die Effektivität vieler der im Folgenden beschriebenen Verfahren wurde daher nicht in Studien geprüft, sondern nur an kleinen Patientengruppen untersucht. Da alle adjuvanten Therapien eine intraepitheliale Persistenz des Virus nicht verhindern können, ist ein bleibender Effekt dieser Therapien fraglich [31].

\section{Interferon}

Die systemische Gabe von Interferon ist nach wie vor die am besten untersuchte adjuvante Therapie. Interferone gehören zu den Zytokinen und werden u. a. bei Virusinfektionen ausgeschüttet. Der genaue Wirkmechanismus ist nicht bekannt, es scheint über multiple molekulare Mechanismen eine Hemmung der viralen Proteinsynthese einzutreten. Typische Nebenwirkungen einer Interferontherapie sind grippeähnliche Symptome mit Kopfund Gliederschmerzen, Fieber und Diarrhoe insbesondere zu Beginn der Therapie, daher ist die Compliance der behandelten Patienten gering. Zum zwingenden Therapieabbruch führen in 10-20\% der Patienten neuropsychiatrische Nebenwirkungen sowie seltener eine Knochenmarksuppression mit Auftreten einer Thrombound Leukopenie.

$\alpha$-Interferon wurde als eines der wenigen Adjuvanzien in den 1980er Jahren im Rahmen einer randomisierten multizentrischen Doppelblindstudie untersucht [32]. Dabei zeigte sich eine dosisabhängige Reduktion des Papillomwachstums mit einem guten Ansprechen bei einem Drittel der Patienten. Der therapeutische Effekt war jedoch nach Absetzen des Medikaments rasch rückläufig (ReboundPhänomen). In weiteren Untersuchungen zeigten sich signifikante Unterschiede im Ansprechen auf die Interferongabe zwischen den verschiedenen HPV-Subtypen. Patienten mit einer HPV-6-Infektion zeigten ein Ansprechen von $64 \%$, während solche mit einer HPV-11-Infektion lediglich zu $14 \%$ auf die Therapie reagierten. 
Aufgrund der multiplen systemischen Nebenwirkungen und der damit assoziierten schlechten Compliance wurde die Interferonbehandlung weitgehend verlassen und findet nur noch gelegentlich bei schweren juvenilen Verläufen Anwendung. Nach internationaler Zulassung sogenannter PEGylierter Interferone mit deutlich verzögerter Wirkstofffreisetzung durch Bindung an Polyethylenglycol muss allerdings das limitierende Nebenwirkungsprofil neu beurteilt werden. Durch konstante Serumspiegel ist der verbesserte therapeutische Effekt bei limitierten Nebenwirkungen insbesondere in der Behandlung der Hepatitis $C$ unterdessen gut belegt. In ersten Behandlungsversuchen scheint sich eine gute Wirksamkeit auch bei RRP zu zeigen [33].

\section{Antirefluxive Therapie $\left(\mathrm{H}_{2}\right.$-Blocker)}

Wissenschaftliche Rationale für eine antirefluxive Therapie von RRP-Patienten ist die Annahme, dass durch den Reflux von Säure und Enzymen eine chronische Entzündung der Larynxschleimhaut entsteht, welche ihrerseits eine bestehende subklinische HPV-Infektion aktivieren kann. Zweiter Effekt einer hochdosierten Therapie mit Cimetidin ist eine Immunmodulation mit Stimulation der Bildung von Th1-Helferzellen (Th1 > Th2-Zell-Shift) und damit eine Inhibition der intrazellulären Virusreplikation. Es gibt Untersuchungen zur antirefluxiven Behandlung insbesondere mit $\mathrm{H}_{2}$-Blockern, welche klinische Erfolge dokumentieren. Insbesondere die postoperative Narbenbildung nach chirurgischer Abtragung scheint sich unter $\mathrm{H}_{2}$-Blockern zu reduzieren [34]. Kontrollierte Studien zu dieser Behandlung stehen jedoch aus.

\section{Indol-3-Carbinol}

Indol-3-Carbinol ist ein natürlicher Inhaltsstoff von grünem Gemüse und moduliert die Enzymaktivität von Cytochrom P450. Weiterhin wurde für die Substanz eine Hochregulation der Apoptose in einigen Krebszelllinien nachgewiesen. Die klinische Wirksamkeit wurde in einer klinischen Untersuchung belegt [35], auch hier fehlen jedoch placebokontrollierte Studien zur Effizienz und Verträglichkeit der Therapie.

\section{Photodynamische Therapie}

Die photodynamische Therapie beruht auf der vermehrten Anreicherung einer lokal oder systemisch verabreichten fotosensibilisierenden Substanz in erkrankter Schleimhaut. Bei der anschließenden Lichttherapie kommt es zur Freisetzung von Sauerstoffradikalen und zum Zelltod. Zur photodynamischen Therapie bei der RRP mit den Photosensibilisatoren Hämatoporphyrin-Derivat ( $\mathrm{HpD}$ ) oder meta-Tetrahydroxyphenylchlorin (mTHPC) gibt es multiple klinisch-experimentelle Arbeiten mit guten Effekten auf die Rezidivrate und die interoperativen Behandlungsintervalle [36], [37]. Risiko der Therapie ist eine lokale Schwellung mit Gefahr der Stenosierung des Atemwegs, kontrollierte Studien fehlen auch hier.

\section{Cidofovir (Vistide)}

Cidofovir ist ein Cytosinanalogon, welches die DNA-Replikation hemmt und zur Therapie der Zytomegalie-induzierten Retinitis bei HIV-Patienten zugelassen ist. Für diese Indikation wird es aufgrund einer signifikanten Nephrotoxizität nur selten verwendet. Das Medikament weist eine potente In-vitro- und In-vivo-Aktivität gegen ein breites Spektrum von Herpesviren, Polyomaviren, Adenoviren, Papillomaviren auf.

Es existieren multiple Untersuchungen zur Off-Label-Anwendung entweder als intraläsionale Injektion oder als systemisch angewandte Therapie bei der RRP. Dabei wurde vielfach ein deutlicher Nutzen der Therapie bis hin zu Berichten von kompletten Remissionen belegt [38], [39]. Problematisch in der Bewertung der Therapie ist eine ausgeprägte Variabilität von Dosierung, Applikationsform und Häufigkeit der Verabreichung. Der Versuch einer wissenschaftlichen Aufarbeitung der bisher publizierten Daten ergab dennoch vielversprechende Ergebnisse mit einer mittleren Ausheilung von $57 \%$ [40].

Die topische oder systemische Applikation von Cidofovir hat nur einen transienten Effekt auf die Hemmung der viralen Genomreplikation von Humanen Papillomaviren. Dies macht eine wiederholte Gabe des Medikaments notwendig. Bei der systemischen Gabe von Cidofovir ist dann jedoch die oben genannte renale Toxizität ein limitierender Faktor in der klinischen Anwendung. Weiterhin zeigt sich im Tierversuch mit Ratten bei der systemischen Gabe die Induktion von Adenokarzinomen [41]. Auch im In-vitroVersuch zeigt sich eine erhöhte Kanzerogenität des Medikaments, sodass die Therapie trotz vielversprechender Ansprechraten kontrovers diskutiert wird [42].

\section{Bevacizumab (Avastin)}

Bei Bevacizumab handelt es sich um einen humanisierten monoklonalen Antikörper, welcher an den Wachstumsfaktor VEGF (Vascular Endothelial Growth Factor) bindet. Dieser ist relevant für die Kapillarisierung von Gewebe, insbesondere gut vaskularisiertes Tumorgewebe. Durch die Blockade von VEGF wirkt Bevacizumab als Angiogenesehemmer und ist als solcher zur Behandlung multipler maligner Tumoren zugelassen. Grundlage der Behandlung von RRP-Patienten mit Bevacizumab ist die ausgeprägte Kapillarisierung der Papillome, welche ebenfalls über die VEGF-Blockade gehemmt werden soll.

Erste klinische Arbeiten zu dieser Therapie zeigen vielversprechende Ansprechraten mit Verlängerung der Therapieintervalle und partiellen sowie zum Teil auch kompletten Remissionen [43], [44]. Der Antikörper wird sowohl lokal injiziert als auch bei schweren Verläufen systemisch verabreicht. Nebenwirkungen sind selten. Auch bei diesem Therapieansatz steht nicht die Elimination des die Erkrankung auslösenden Papillomavirus, sondern die Entstehung 
der papillomatösen Läsionen im Fokus. Langzeitergebnisse und kontrollierte Studien bleiben auch hier abzuwarten.

\section{Therapeutische Vakzinierung}

Die Impfung gegen humane Papillomaviren stellt einen effektiven präventiven Ansatz zur Vermeidung einer Infektion dar. Da bei vermuteter Übertragung des Virus im Geburtskanal präventive Effekte erst in der nachfolgenden Generation zu erwarten sind, wird die Wirkung der Vakzinierung derzeit auch im Kontext einer immunologischen Therapie untersucht. Derzeit sind 2 Impfstoffe zur Prävention einer HPV-Infektion des Genitaltrakts zugelassen:

- Der bivalente Impfstoff Cervarix wirkt gegen das L1Kapsidprotein der HPV-Typen 16 und 18, welche als High-Risk-Virustypen in der Pathogenese des Zervixkarzinoms relevant sind, als Auslöser der RRP aber nur eine untergeordnete Rolle haben.

- Der tetravalente Impfstoff Gardasil richtet sich gegen die HPV-Typen 6, 11, 16 und 18 und erfasst präventiv somit auch die bei der RRP wesentlichen Typen 6 und 11. Der Impfstoff enthält synthetisch hergestellte Virushüllen (sog. Virus-like Particles), welche eine immunologische Reaktion auf das L1-Hüllprotein induzieren.

Als wirksamer Mechanismus einer therapeutischen Vakzinierung ist die Vermeidung einer Infektion bislang nicht infizierten Gewebes denkbar, aber auch eine immunologische Reaktion im Bereich infizierter Schleimhaut ist möglich. Erste Kasuistiken zeigen differente Ergebnisse, in einigen Untersuchungen wird eine deutliche Verlängerung der interoperativen Intervalle beschrieben [45], [46], [47]. Vorteil ist die geringe Rate an Nebenwirkungen, weshalb die Impfung als Therapieversuch großzügig indiziert wird.

\section{Merke}

Nur wenige der adjuvanten Therapien der RRP sind in kontrollierten klinischen Studien geprüft, keine Therapie ist für diese Indikation zugelassen. Dennoch existieren zu vielen Therapieformen vielversprechende klinische Daten. Insbesondere bei schweren Krankheitsverläufen kann eine adjuvante Therapie erwogen werden.

\section{Ausblick und Schlussfolgerung}

Mit Zulassung der HPV-Vakzine in Deutschland zunächst für Mädchen, seit 2018 auch für Jungen als Leistung der gesetzlichen Krankenkassen besteht die berechtigte Hoffnung auf eine deutliche Reduktion der Inzidenz HPV-induzierter Erkrankungen. Bereits im Jahr 2007 wurde in Australien die Impfung gegen HPV flächendeckend und mit guter Akzeptanz eingeführt. Epidemiologischen Studien zufolge könnte durch diese Maßnahme die jährliche Neuerkrankungsrate an Zervixkarzinomen deutlich reduziert werden [48]. Erste Daten zur Inzidenz der RRP belegen, dass auch diese nach Einführung der Impfung jährlich rück- läufig ist [49], sodass zukünftig möglicherweise die Therapie der RRP zugunsten der Prophylaxe in den Hintergrund treten wird.

Die RRP stellt aufgrund des variablen und rekurrenten Verlaufs mit der Notwendigkeit einer häufigen operativen Therapie eine therapeutische Herausforderung dar. Die Entwicklung verschiedener Hilfsmittel zur chirurgischen Therapie schafft neue Ansätze zur verbesserten Kontrolle der Erkrankung mit geringstmöglicher Schädigung der anatomischen Strukturen und Verminderung von iatrogenen funktionellen Einbußen bei den betroffenen Patienten. Das Fehlen von kontrollierten Studien limitiert die Einschätzung der zur Verfügung stehenden adjuvanten Therapien. Dennoch bieten sich gerade bei schweren Verläufen mit fehlender Kontrolle der Erkrankung durch chirurgische Therapieansätze multiple Möglichkeiten mit vielversprechenden adjuvanten Therapeutika.

\section{KERNAUSSAGEN}

- Die rezidivierende respiratorische Papillomatose (RRP) ist eine durch humane Papillomaviren verursachte Erkrankung, deren Infektionsweg nicht zweifelsfrei geklärt ist.

- Man unterscheidet abhängig vom Lebensalter der Erstmanifestation die juvenile (JORRP) von der adulten (AORRP) Form, wobei erstere hochgradigere Verläufe aufweist.

- Der klinische Verlauf der Erkrankung ist sehr variabel und abhängig vom nachgewiesenen Virustyp.

- Grundlegendes Therapieprinzip ist die schonende chirurgische Abtragung zur Kontrolle der Erkrankung sowie zum funktionellen Erhalt des Atem- und Schluckwegs. Radikal-chirurgische Ansätze sind aufgrund der Viruspersistenz im umgebenden Gewebe nicht angezeigt.

- Eine medikamentös-adjuvante Therapie ist insbesondere bei schwergradigen Verläufen mit hoher Therapiefrequenz indiziert. Hierfür stehen verschiedene Medikamente zur Verfügung, welche jedoch weder für die Indikation zugelassen noch in kontrollierten Studien untersucht worden sind. Dennoch existieren hoffnungsvolle klinisch-experimentelle Untersuchungen, insbesondere für die Präparate Cidofovir und Bevacizumab.

Interessenkonflikt

Dr. Christine Langer erhielt ein Honorar für Vortragstätigkeit/Expertenforum der Fa. MSD Sharp \& Dome GmbH. 


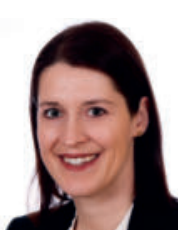

\section{Christine Langer}

Dr. med., 1996-2003 Studium der Humanmedizin an der Georg-August-Universität Göttingen und an der Justus-Liebig-Universität Gießen, 2003-2008 Facharztausbildung für Hals-Nasen-Ohrenheilkunde, 2010 Fachweiterbildung Medikamentöse Tumortherapie, 2013 Fachweiterbildung Plastische Operationen, seit 2009 Oberärztin an der HNO-Universitätsklinik Gießen. Schwerpunkte: Kopf-Hals-Onkologie, plastisch-rekonstruktive Chirurgie.

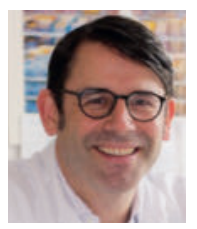

\section{Claus Wittekindt}

Prof. Dr. med., 1992-1998 Studium der Humanmedizin an der Universität zu Köln, 1998-2003 Facharztausbildung für Hals-, Nasen- und Ohrenheilkunde am Universitätsklinikum Köln, 2003-2006 Tätigkeit als Facharzt an der HNO-Klinik Köln, 20062010 Oberarzt an der HNO-Universitätsklinik Jena, 2010-2018 Leitender Oberarzt Universitätsklinik Gießen, seit 2018 kommissarischer Leiter der HNO-Universitätsklinik Gießen.

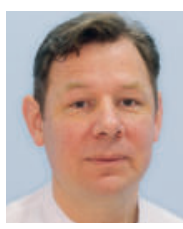

\section{Gregor Wolf}

Dr. med., 1997-2004 Studium der Humanmedizin an der Universität des Saarlands, 2005-2010 Facharztausbildung für Hals-, Nasen- und Ohrenheilkunde an den Universitätsklinika Erlangen und Homburg/ Saar, 2010-2017 Oberarzt Universitätsklinik Homburg/Saar, seit 2018 Leitender Oberarzt HNO-Universitätsklinikum Gießen.

\section{Korrespondenzadresse}

\section{Dr. med. Christine Langer}

Klinik für HNO-Heilkunde, Kopf-/Halschirurgie,

Plastische Operationen

Universitätsklinikum Gießen und Marburg,

Standort Gießen

Justus-Liebig-Universität Gießen

Klinikstraße 33

D-35392 Gießen

E-Mail: christine.langer@hno.med.uni-giessen.de

Wissenschaftlich verantwortlich gemäß Zertifizierungsbestimmungen

Wissenschaftlich verantwortlich gemäß Zertifizierungsbestimmungen für diesen Beitrag ist Dr. med. Christine Langer, Gießen.

\section{Literatur}

[1] Larson DA, Derkay CS. Epidemiology of recurrent respiratory papillomatosis. APMIS 2010; 118: 450-454

[2] Gallagher TQ, Derkay CS. Pharmacotherapy of recurrent respiratory papillomatosis: an expert opinion. Expert Opin Pharmacother 2009; 10: 645-655

[3] Lindeberg H, Elbrond O. Laryngeal papillomas: the epidemiology in a Danish subpopulation 1965-1984. Clin Otolaryngol Allied Sci 1990; 15 (2): 125-131

[4] Derkay CS, Wiatrak B. Recurrent respiratory papillomatosis: a review. Laryngoscope 2008; 118: 236-1247

[5] Buchinsky F], Donfack J. Age of child, more than HPV type, is associated with clinical course in recurrent respiratory papillomatosis. PLoS ONE 2008; 28: 2263

[6] Armstrong LR, Derkay CS, Reeves WC. Initial results from the national registry for juvenile-onset recurrent respiratory papillomatosis. RRP Task Force. Arch Otolaryngol Head Neck Surg 1999; 125 (7): 743-748

[7] Munoz N, Bosch FX, de Sanjosé S. Epidemiologic classification of human papillomavirus types associated with cervical cancer. N Engl J Med 2003; 348: 518-552

[8] Rabah R, Lancaster WD, Thomas R, Gregoire L. Human papillomavirus-11-associated recurrent respiratory papillomatosis is more aggressive than human papillomavirus-6-associated disease. Pediatr Dev Pathol 2001; 4 (1): 68-72

[9] Kashima HK, Kessis T, Mounts P, Shah K. Polymerase chain reaction identification of human papillomavirus DNA in CO2 laser plume from recurrent respiratory papillomatosis. Otolaryngol Head Neck Surg 1991; 104 (2): 191-195

[10] Kashima H, Wu TC, Mounts P et al. Carcinoma ex-papilloma: histologic and virologic studies in whole-organ sections of the larynx. Laryngoscope 1988; 98 (6 Pt 1): 619-624

[11] Yoshpe NS. Oral and laryngeal papilloma: a pediatric manifestation of sexually transmitted disease? Int J Pediatr Otorhinolaryngol 1995; 31 (1): 77-83

[12] Fredericks BD, Balkin A, Daniel HW et al. Transmission of human papillomaviruses from mother to child. Aust N Z J Obstet Gynaecol 1993; 33 (1): 30-32

[13] McKenna M, Brodsky L. Extraesophageal acid reflux and recurrent respiratory papilloma in children. Int J Pediatr Otorhinolaryngol 2005; 69 (5): 597-605

[14] Shah KV, Stern WF, Shah FK et al. Risk factors for juvenile onset recurrent respiratory papillomatosis. Pediatr Infect Dis 1998; 17 (5): 372-376

[15] Larson DA, Derkay CS. Epidemiology of recurrent respiratory papillomatosis. APMIS 2010; 118: 450-454

[16] Kashima H, Shah F, Lyles A. A comparison of risk factors in juvenile-onset and adult-onset respiratory papillomas. Laryngoscope 1992; 102: 9-13

[17] Derkay CS. Recurrent respiratory papillomatosis. Laryngoscope 2001; 111: 57-69

[18] Reeves WC, Ruparelia SS, Swanson KI et al. National registry for juvenile-onset recurrent respiratory papillomatosis. Arch Otolaryngol Head Neck Surg 2003; 129 (9): 976-982

[19] Silver RD, Rimell FL, Adams GL. Diagnosis and management of pulmonary metastasis from recurrent respiratory papillomatosis. Otolaryngol Head Neck Surg 2003; 129: 622-629

[20] Abramson AL, Nouri M, Mullooly V et al. Latent Human Papillomavirus infection is comparable in the larynx and trachea. J Med Virol 2004; 72 (3): 473-477 
[21] Leunig A, Betz CS, Mehlmann M. A pilot serie demonstrating fluorescence staining of laryngeal papilloma using 5-aminolevulinic acid. Laryngoscope 2000; 110: 1783-1785

[22] Derkay CS, Hester RP, Burke P et al. Analysis of a staging assessment system for prediction of surgical interval in recurrent respiratory papillomatosis. Int J Pediatr Otorhinolaryngol 2004; 68 (12): 1493-1498

[23] Reeves WC, Ruparelia SS, Swanson KI et al. National registry for juvenile-onset recurrent respiratory papillomatosis. Arch Otolaryngol Head Neck Surg 2003; 129 (9): 976-982

[24] Tasca RA, McCormick M, Clarke RW. British Association of Paediatric Otorhinolaryngology members experience with recurrent respiratory papillomatosis. Int J Pediatr Otorhinolaryngol 2006; 70 (7): 1183-1187

[25] Pasquale K, Wiatrak B, Woolley A, Lewis L. Microdebrider versus CO2 laser removal of recurrent respiratory papillomas: a prospective analysis. Laryngoscope 2003; 113 (1): 139-143

[26] Schraff S, Derkay CS, Burke B, Lawson L. American Society of Pediatric Otolaryngology members' experience with recurrent respiratory papillomatosis and the use of adjuvant therapy. Arch Otolaryngol Head Neck Surg 2004; 130 (9): 1039-1042

[27] Andratschke A, Betz C, Leunig A. Larynxpapillomatose, Ätiologie, Diagnostik und Therapie. HNO 2008; 56 (12): 1190-1196

[28] Johnson K, Derkay C. Palliative aspects of recurrent respiratory papillomatosis. Otolaryngol Clin North Am 2009; 42 (1): $57-70$

[29] Kashima HK, Kessis T, Mounts P, Shah K. Polymerase chain reaction identification of human papillomavirus DNA in CO2 laser plume from recurrent respiratory papillomatosis. Otolaryngol Head Neck Surg 1991; 104 (2): 191-195

[30] Hartnick C], Boseley ME, Franco RA et al. Efficacy of treating children with anterior commissure and true vocal fold respiratory papilloma with the 585 -nm pulsed-dye laser. Arch Otolaryngol Head Neck Surg 2007; 133: 127-130

[31] Carifi M, Napolitano D, Morandi M, Dall'Olio D. Recurrent respiratory papillomatosis: current and future perspectives. Ther Clin Risk Manag 2015; 11: 731-738

[32] Healy GB, Gelber RD, Trowbridge AL et al. Treatment of recurrent respiratory papillomatosis with human leukocyte interferon. Results of a multicenter randomized clinical trial. N Engl J Med 1988, 319: 401-407

[33] Maunsell R, Bellomo-Brandao AM. Pegylated interferon for treating severe recurrent respiratory papillomatosis in a child. A case report. Sao Paolo Med J 2018; 136 (4): 376-381

[34] Holland B, Koufman J, Postma G, McGuirt W. Laryngopharyngeal reflux and laryngeal web formation in patients with pediatric recurrent respiratory papillomas. Laryngoscope 2002; 112: 1926-1929

[35] Rosen CA, Bryson PC. Indole-3-carbinol for recurrent respiratory papillomatosis: long-term results. J Voice 2004, 18: $248-253$

[36] Abramson AL, Shikowitz M], Mullooly VM et al. Variable light-dose effect on photodynamic therapy for laryngeal papillomas. Arch Otolaryngol Head Neck Surg 1994; 120 (8): 852-855

[37] Shikowitz M], Abramson AL, Steinberg BM et al. Clinical trial of photodynamic therapy with meso-tetra (hydroxyphenyl) chlorin for respiratory papillomatosis. Arch Otolaryngol Head Neck Surg 2004; 131 (2): 99-105

[38] Pudzuhn A, Welzel C, Bloching M, Neumann K. Intralesional cidofovir application in recurrent laryngeal papillomatosis. Eur Arch Otorhinolaryngol 2007; 264: 63-70
[39] Pransky SM, Albright JT, Magit AE. Long-term follow-up of pediatric recurrent respiratory papillomatosis managed with intralesional cidofovir. Laryngoscope 2003; 113: 1583-1587

[40] Chadha NK, James AL. Antiviral agents for the treatment of recurrent respiratory papillomatosis: a systematic review of the English language literature. Otolaryngol Head Neck Surg 2007; 136: 863-869

[41] Bedard J, May S, Lis M et al. Comparative study of the anti-human cytomegalovirus activities and toxicities of a tetrahydrofuran phosphonate analogue of guanosine and cidofovir. Antimicrob Agents Chemother 1999; 43: 557-567

[42] Nawka T. Larynxpapillomatose. Laryngorhinootologie 2018; 97 (10): 670-671

[43] Best SR, Mohr M, Zur KB. Systemic bevcizumab for recurrent respiratory papillomatosis: A national survey. Laryngoscope 2017; 127 (10): 2225-2229

[44] Sidell DR, Nassar M, Cotton RT et al. High-dose sublesional bevacizumab (avastin) for pediatric recurrent respiratory papillomatosis. Ann Otol Rhinol Laryngol 2014; 123 (3): 214-221

[45] Yiu Y, Fayson S, Smith H, Matrka L. Implementation of Routine HPV Vaccination in the Management of Recurrent respiratory Papillomatosis. Ann Otol Rhinol Laryngol 2019; 128 (4): 309-315

[46] Papaioannou VA, Arens C. Behandlungsergebnisse der rezidivierenden respiratorischen Papillomatose mit Gardasil. Laryngorhinootologie 2018: 97 (S02): 213

[47] Papaioannou VA, Lux A, Zimmermann S, Arens C. Behandlungsergebnisse bei rezidivierender respiratorischer Papillomatose - retrospektive Analyse juvenile und adulter Fälle. HNO 2017; 65 (11): 923-932

[48] Hall MT, Simms KT, Lew JB et al. The projected timeframe until cervical cancer elimination in Australia: a modelling study. Lancet Public Health 2019; 4 (1): e19-e27

[49] Novakovic D, Cheng ATL, Zurynski Y et al. A Prospective Study of the Incidence of Juvenile-Onset Recurrent Respiratory Papillomatosis After Implementation of a National HPV Vaccination Program. J Infect Dis 2018; 217 (2): 208-212

Bibliografie

DOI https://doi.org/10.1055/a-0896-9729 Laryngo-Rhino-Otol 2019; 98: 577-587 (c) Georg Thieme Verlag KG Stuttgart · New York ISSN 0935-8943 


\section{Punkte sammeln auf CME.thieme.de}

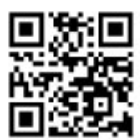

Diese Fortbildungseinheit ist in der Regel 12 Monate online für die Teilnahme verfügbar.

Den genauen Einsendeschluss finden Sie unter https://eref.thieme.de/CXDZ9BN.

Sollten Sie Fragen zur Online-Teilnahme haben, finden Sie unter https://cme.thieme.de/hilfe eine ausführliche Anleitung. Wir wünschen viel Erfolg beim Beantworten der Fragen!

Unter https://eref.thieme.de/CXDZ9BN oder über den QR-Code kommen Sie direkt zur Startseite des Wissenstests.

VNR 2760512019156640219

\section{Frage 1}

Wann spricht man von der juvenilen Form der RRP (JORPP)?

A bei einem bevorzugten Befall des Naso- und Oropharynx

B bei einem Auftreten vor dem 12. Lebensjahr

C beim Auftreten der ersten klinischen Symptome im 1. bis 3. Lebensjahr

D beim Vorliegen von Begleitsymptomen wie Gedeihstörungen oder Dysphagie

E beim Nachweis einer genitalen HPV-Infektion der Mutter

\section{Frage 2}

Welche Aussage zur medikamentösen adjuvanten Therapie der RRP trifft zu?

A Bevacizumab wirkt über eine Aktivierung der T-Zellen.

B Cidofovir ist ein Angiogenesehemmer.

C Der limitierende Faktor bei der systemischen Gabe von Cidofovir ist die Nephrotoxizität.

D Antirefluxive $\mathrm{H}_{2}$-Blocker wirken über einen direkten virotoxischen Effekt.

E Interferon wird bei der AORPP standardmäßig als Adjuvans verabreicht.

\section{Frage 3}

Welche Aussage zum Infektionsweg der RRP trifft nicht zu?

A Es wird eine vertikale Übertragung im Geburtskanal angenommen.

B JORRP-Patienten haben eine erhöhte Prävalenz von maternalen anogenitalen Papillomen.

C JORPP-Patienten werden überproportional häufig vaginal entbunden.

D Bei Neugeborenen von Müttern mit genitalen Kondylomen kann HPV mehrere Wochen lang in der pharyngealen Schleimhaut nachgewiesen werden.

E Bei maternalen genitalen Kondylomen tritt postpartal bei über 50 \% der Neugeborenen eine RRP auf.

\section{Frage 4}

Welche Aussage zum Humanen Papillomavirus trifft zu?

A Es handelt sich um ein Virus aus der Gruppe der Enteroviridae.

B Humane Papillomaviren sind artübergreifend übertragbar.

C Häufigste Erreger der RRP sind HPV 6 und 11.

D HPV 16 und 18 verursachen milde Verläufe der RRP.

E Eine Infektion mit HPV betrifft insbesondere subepitheliale Strukturen.

\section{Frage 5}

Welche Antwort ist richtig? Der klinische Verlauf der RRP ist...

A unabhängig vom Alter der Erstmanifestation.

B abhängig von den Komorbiditäten des Patienten.

$C$ bezogen auf die Häufigkeit der notwendigen Eingriffe vorhersehbar.

D häufig aggressiver beim Nachweis von HPV 11 als beim Nachweis von HPV 6.

E unabhängig von einer medikamentös-adjuvanten Therapie.

\section{Frage 6}

Welche Antwort ist falsch? Cidofovir...

A ist ein Cytosinanalogon.

B wird intraläsional oder systemisch verabreicht.

$C$ ist zur Behandlung der HPV-Infektion zugelassen.

D wurde ursprünglich zur Therapie der HIV-assoziierten CMVRetinitis entwickelt.

E hat im In-vitro-Versuch kanzerogene Eigenschaften. 


\section{Punkte sammeln auf CME.thieme.de}

\section{Fortsetzung $\ldots$}

\section{Frage 7}

Eine der folgenden Therapien gehört nicht zu den häufig verwendeten medikamentösen adjuvanten Therapien der RRP. Welche?
A Nivolumab
B Bevacizumab
C Cidofovir
D Interferon
E $\mathrm{H}_{2}$-Blocker

\section{Frage 8}

Welche Aussage zur chirurgischen Behandlung der RRP mit dem $\mathrm{CO}_{2}$-Laser trifft nicht zu?

A Es kommt zu einer Verdampfung von Gewebe.

B Es kann zur thermischen Schädigung von gesundem Gewebe kommen.

C Eine Vaporisation von Viruspartikeln kann auftreten.

D Eine Übertragung des Virus auf andere durch Aerosole ist ausgeschlossen.

E Es müssen Schutzmaßnahmen zur Infektionsprophylaxe im Operationssaal getroffen werden.

\section{Frage 9}

Welche Aussage zur Verwendung des Microdebriders bei der Therapie der RRP ist nicht korrekt?

A Es tritt keine Vaporisation von Viruspartikeln auf.

B Eine Schädigung von gesundem Gewebe kann bei dieser Therapie nicht auftreten.

C Das abzutragende Gewebe wird angesaugt.

D Der Microdebrider arbeitet mit rotierenden Klingen.

E Es handelt sich um das am häufigsten verwendete Verfahren im angloamerikanischen Sprachraum.

\section{Frage 10}

Was sind die therapeutischen Ziele bei der Behandlung der RRP? Welche Aussage trifft nicht zu?
A Freihalten des Atem- und Schlucktrakts
B Funktionserhalt des Kehlkopfs
C Vermeidung von Narben und Synechien endolaryngeal
D radikale Abtragung des gesamten HPV-infizierten Gewebes
E Verlängerung der interoperativen Intervalle 